\title{
Investigation of Magnetic Flux Distribution of EV Wireless Charging Systems
}

\author{
Chirag Panchal ${ }^{1}$, Junwei Lu ${ }^{1}$, Sascha Stegen ${ }^{1}$ \\ ${ }^{1}$ Griffith School of Engineering, Griffith University, Nathan Campus, Brisbane, Australia 4111 \\ ${ }^{1} \mathrm{c}$. panchalegriffith.edu.au \\ ${ }^{1} j$.luegriffith.edu. au \\ ${ }^{1}$ s.stegen@griffith.edu. au
}

\begin{abstract}
A High Frequency and High Power Density Wireless Planar Transformer (HFHPD-WPT) is a very attractive future technology which can provide power to an electric vehicle (EV) through a contactless charging system. In this paper, EMC computer modeling and simulation techniques are employed to investigate the magnetic flux distribution and associated EMC problems such as stray fluxes and hot spots. The bifilar planar spiral coils of the transformer are designed on multilayer PCB. A finite element method (FEM) has been used to calculate the magnetic field. The effect of the planar magnetic ferrite cores on magnetic flux distribution has been investigated by using three designs, where the first design has the ferrite core only at the primary side, the second draft has a planar core on the primary and secondary side, and the third design has a U-Shape magnetic core for the primary and secondary side. Both designs are operating at a resonant frequency of $60 \mathrm{kHz}$. A new proposed design is introduced to minimize flux leakages and reduce hot spots, in order to improve the flux distribution and to increase the magnetizing impedance.
\end{abstract}

Keywords - Bifilar winding, Electromagnetic Compatibility (EMC), Finite element method (FEM), High Frequency and High Power Density Wireless Planar Transformer (HFHPD-WPT), Wireless Battery Charging.

\section{INTRODUCTION}

W ireless charging systems have become one of the famous and innovative technologies in the last decade for various applications including wireless biomedical devices, compact electronic products such as smart phones, and also concepts for electrical vehicles (EVs) [1-4]. The most electronics devices are usually charged by conventional battery chargers through wired power adaptors and they do not have common charger because of different power ratings as well as a missing standard for a common connection plug. This results in the increase of the amount of toxic and nonbiodegradable electronic waste [5-9]. To solve these difficulties, high frequency High Power Density wireless planar transformers (HFHPD-WPTs) can be used in a wireless charging system, to provide an universal and environmental friendly charging solution. There are many types of winding structures such as hexagonal, fractal, circular and rectangular designs that used to charge compact electronics products without any wired connection [10-12]. With the help of inductive coupling, the energy transfer from the primary side to secondary side without any contact which helps to solve contact failure, become more convenient forming a common charging platform for electronic products [5-7].

However, earlier designs are suffering from high losses due to large air gaps and because the coupling coefficient $K$ is smaller in value than unity, so that the leakage inductance value is almost the same as the magnetizing impedance. Additionally, EMC problems in non-efficient designs are created because of high flux leakages. These problems cause lower power transfer and produce possible high powered stray flux which can have significant impact on human health [13].

A basic arrangement of a wireless charging system for Electric Vehicle (EV) is shown in the Fig.1. Before designing the efficient wireless charging system the LLC resonant circuit [14], contactless charging coils and rectifier including filters are three major components that should be considered.

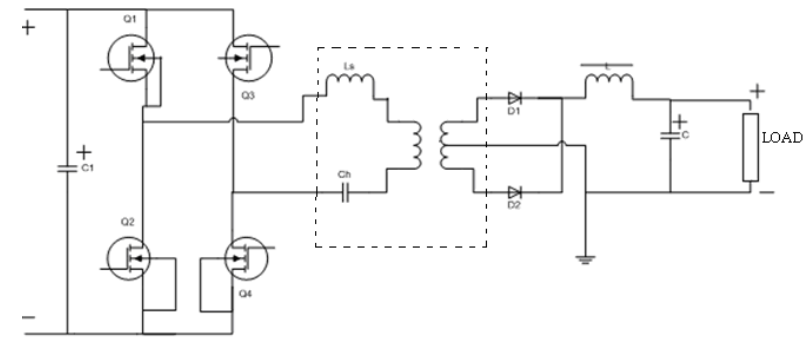

Fig. 1 Basic schematic diagram of wireless charging system including full bridge (High voltage)

Particularly the charging transformer configuration including air gaps, external and internal produced electromagnetic interferences as well as structural errors need to be eliminated in order to produce an efficient system. As discussed in a previous paper [15], the air gap optimization was done at frequency of $60 \mathrm{kHz}$ with a primary-secondarycoil-distance of $14.5 \mathrm{~mm}$ for a maximum power transfer. These optimized values are utilized to increase the power transfer efficiency by using different structured magnetic 
ferrite cores. In addition, the core can not only increase the efficiency but also minimizes flux leakages and avoids EMC problems.

\section{DESIGN CONFIGURATION OF HFHPD-WPT FOR EV}

Initially three configuration cases are discussed. The first configuration contains no ferrite core at the secondary side. The second model is using a planar ferrite core on both, primary and the secondary side. The third design investigates the power distribution of a ferrite core on the primary and secondary side of the transformer which is over lapping the coils, in order to avoid stray losses and hot spots at the magnetic core edges. Fig.2(A) shows a schematic diagram of wireless charging system for an electrical vehicle where the planar ferrite cores are fitted only at the primary side in underground structure while the secondary winding installed underneath of the car without any magnetic ferrite cores. For the second configuration, the primary winding with planar ferrite core is installed in the ground and the secondary coil mounted directly underneath of a car. The schematic arrangement of this wireless charging configuration is presented in Fig.2 (B).

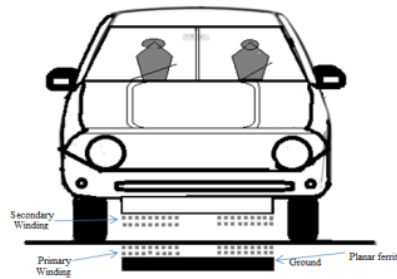

(A)

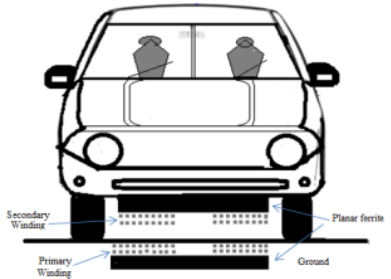

(B)
Fig.2 A schematic of wireless charging system for EV (A) with planar ferrite core at primary winding only (B) both windings with magnetic core

In the final case, a new proposed design arrangement of the wireless charging system is shown in Fig.3, where two Ushaped cores are utilized on the car and on the ground. The idea behind this design is to minimize the leakage flux that appears on the edges of the coils, which can create EMC problems. The U-shaped edges capture the magnetic flux and divert it to the secondary winding to increase the magnetizing impedance of the system. Consequently, the secondary coil has a higher magnetic flux density than the other designs.

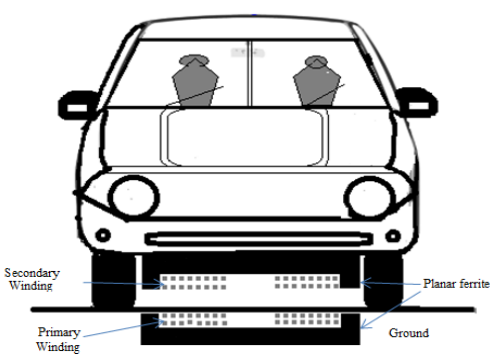

Fig. 3 Wireless charging system for EV with $U$ shaped edge planar ferrite core

A bifilar concept is used to design the primary and secondary coil on FR4 PCB. The primary coil has total 40 turns whereas the secondary has 50 turns. So that the voltage ratio is $1: 1.25$. The outer and inner diameter of the primary coil is $108 \mathrm{~mm}$ and $49 \mathrm{~mm}$, respectively. However, the secondary coil's shape is chosen in rectangular to increase contact surface area and reduce eddy currents by applying round corners. In this structure, the outer length and width are $99 \times 99 \mathrm{~mm}$ whereas the inner space is $25 \times 13 \mathrm{~mm}$ and the space between two coils is $1.6 \mathrm{~mm}$ and trace width is set at $0.5 \mathrm{~mm}$.

\section{MAGNETIC FILED MEASUREMENT AND ANALYSIS}

\section{A. Experimental results of HFHPD-WPT prototypes}

The near field measurement of the HFHPD-WPT using a near field scanner and probes is presented in Fig.3 (A) and (B). In Fig.3 (A), the result is measured when the planar ferrite core is only used on the primary side and the secondary does not have a magnetic core. The magnetic field in this arrangement is around 10 to $15 \mathrm{mV}$ in the center area of the coils because the magnetic fluxes pass through hollow area and is released in the air.

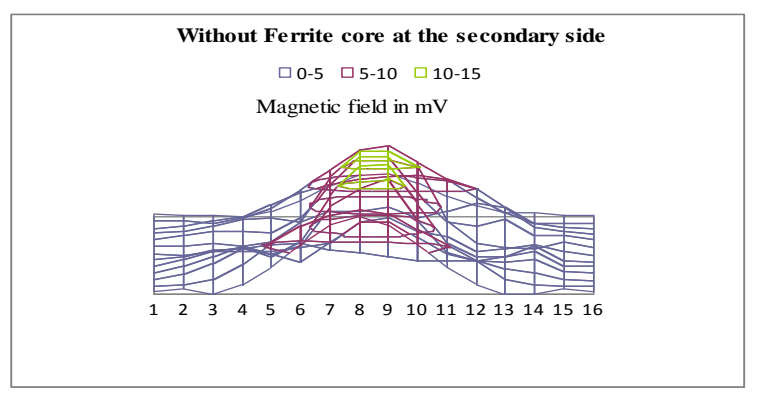

(A)

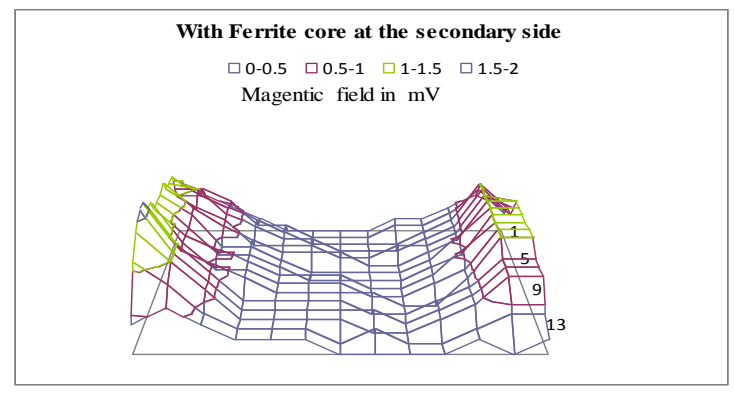

(B)

Fig.5 Near field measurement of HFWPT (A) with magnetic ferrite on the primary side only (B) both sides

However, the near field measurement is carried out when the magnetic cores, that covers the entire coil area, are used on both sides. The measured result is demonstrated in Fig. 3(B) where the magnetic field is 1 to $1.5 \mathrm{mV}$ at the edge due to leakages. Unlike, the center area has no magnetic flux distribution outside the core. This means that the losses are very low if the ferrite is used.

\section{B. Low frequency model's magnetic field analysis using FEM}

In wireless charging systems, the magnetic field and the eddy current distribution for planar spiral coils can be 
determined by the following vector magnetic potential equation (1).

$$
\nabla \times(\nu \nabla \times A)+\sigma(\partial A / \partial t)=J .
$$

where $\mathrm{A}$ is the magnetic vector potential, and $\mathrm{J}$ is the current density, $v$ and $\sigma$ are the magnetic reluctivity, and the conductivity respectively. With the help of linear techniques, the energy function is simplified. Galerkin's method is employed to discretise the governing equation to resolve the problem. The following basic equation of the system matrix can be obtained is presented in equation (2).

$$
\mathrm{G}=[\mathrm{S}]\{\mathrm{A}\}+[\mathrm{M}]\{\mathrm{A}\}-\{\mathrm{K}\}
$$

where the matrix $[\mathrm{S}]$ and $[\mathrm{M}]$ are a global coefficient matrix and the time harmonic matrix respectively. $[\mathrm{K}]$ is the matrix of current source.

\section{Simulation results}

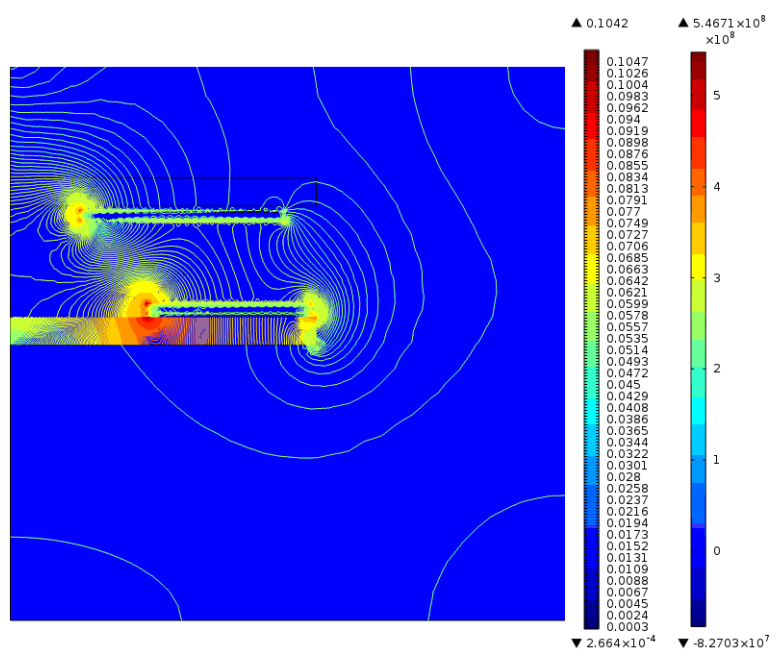

Fig. 4 Magnetic field density and current density of the HFHPD-WPT prototype without ferrite core at secondary side, non-load condition

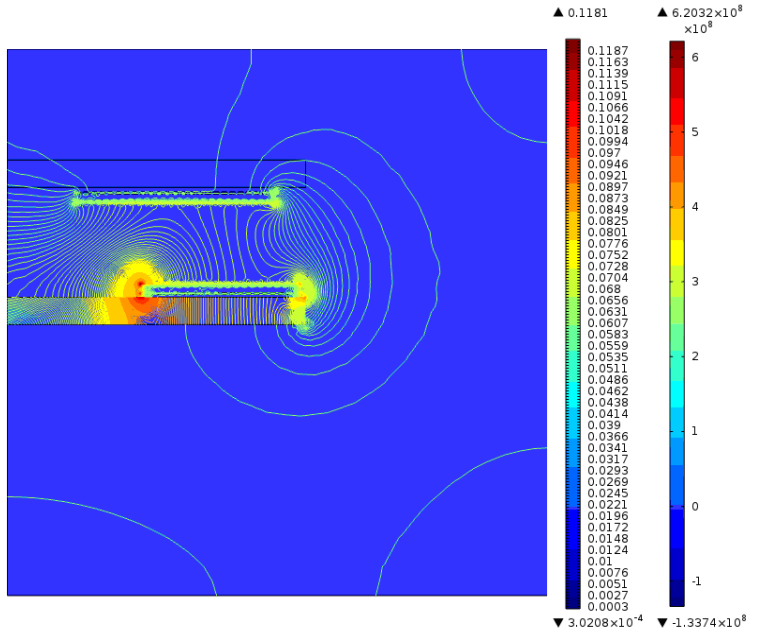

Fig. 5 Magnetic field density and current density of the HFHPD-WPT prototype without ferrite core at secondary side, load condition

In a wireless charging system for $\mathrm{EV}$, planar magnetic ferrites have a significant effect on magnetic flux distribution and flux leakages are investigated by simulation method at the resonant frequency of $60 \mathrm{kHz}$. Fig. 4 and 5 shows magnetic field density and current density of planar spiral coil in an axisymmetric structure when the planar magnetic ferrite core is installed at primary side only. Here, the length of the ferrite is the same as the outer boundary of the coil. In Fig.4, the analysis is being done in no-load condition while the full-load condition is presented in Fig.5. In this case, the magnetic fluxes are not only received by the secondary winding but it also goes at the edges and outside the ferrite core and in air. As a result, it generates eddy current and flux leakages.

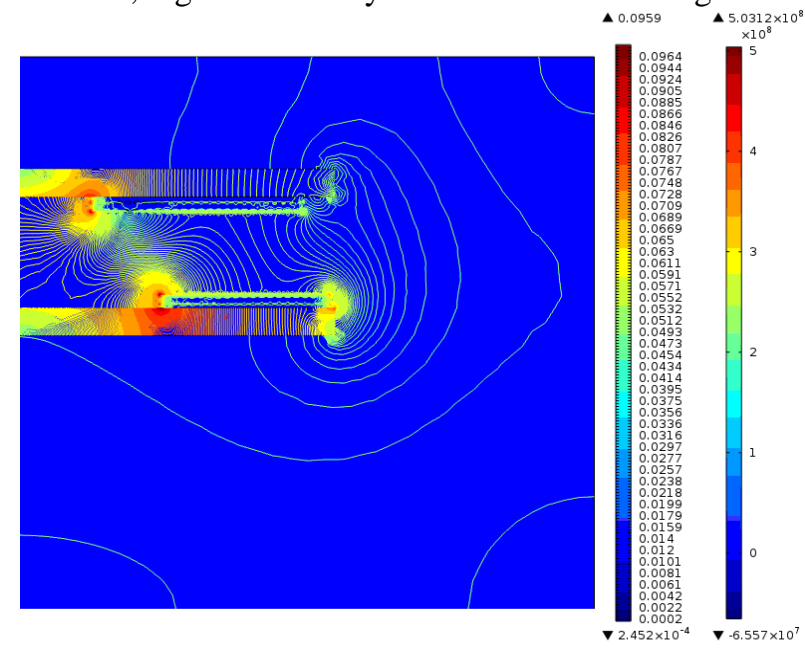

Fig. 6 Magnetic field density and current density of the HFHPD-WPT prototype with ferrite core on both sides, non-load condition

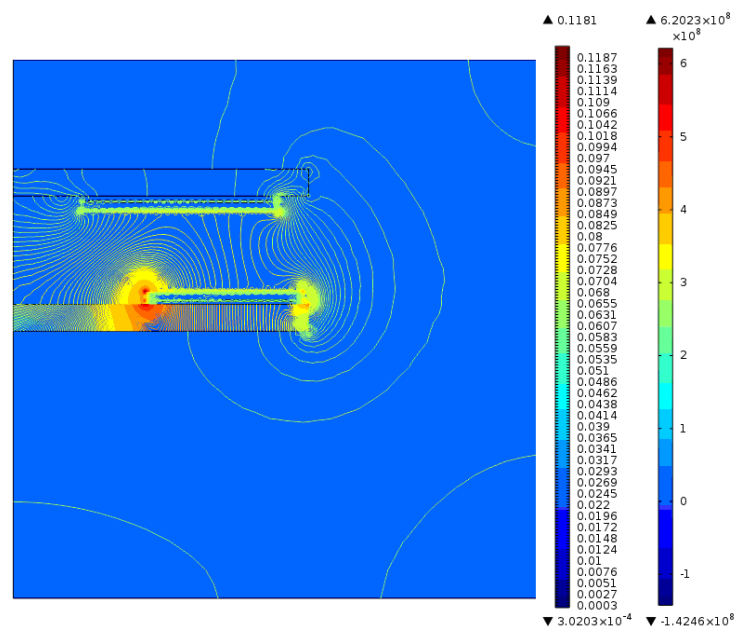

Fig. 7 Magnetic field density and current density of the HFHPD-WPT prototype without ferrite core on both sides, load condition

In Fig 6 and 7, the ferrite core is installed on the primary winding as well as the secondary winding is mounted underneath of the car. Fig.6 illustrates computational analysis result in no-load condition, while the full-load condition is demonstrated in Fig.7. In this arrangement, the magnetic fluxes which generates eddy current and flux leakages are stopped by the ferrite core at the secondary winding. 
In the above designs, the magnetic fluxes which leakages at the secondary side are solved but the flux leakages at the edges still causes a problem because of EMC consideration and generating hot spots. To alleviate this problem, a new proposed design is presented in Fig. 8 and 9 in no-load and full-load conditions respectively. The rectangle edges of the ferrite stops flux leakages at the edges and minimize the eddy current losses. They also divert the magnetic fluxes inside the core and to the secondary side. This result in improvement of the power flux distribution and lighten EMC problems.

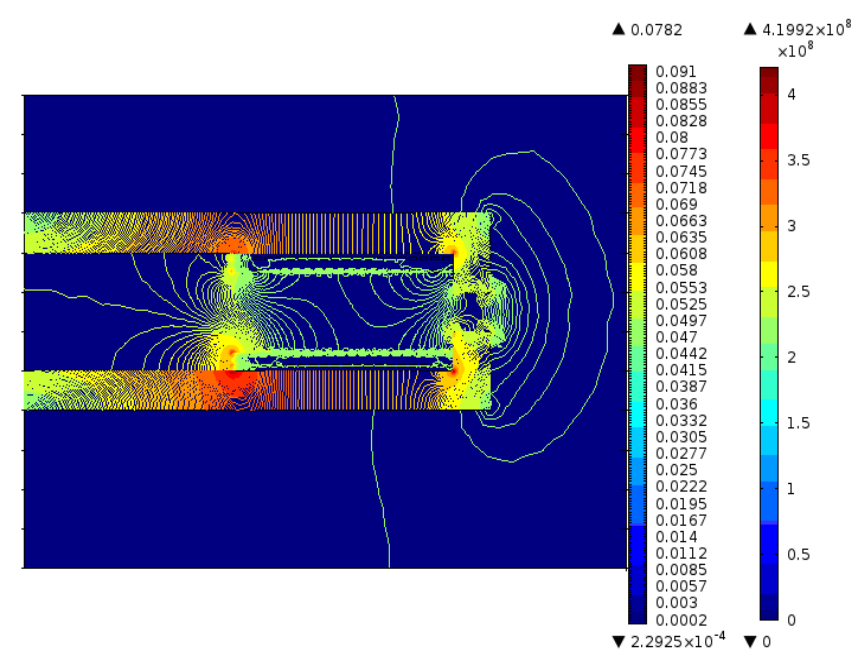

Fig. 8 Magnetic field density and current density of the HFHPD-WPT prototype with U-shaped ferrite cores on both sides, no-load condition

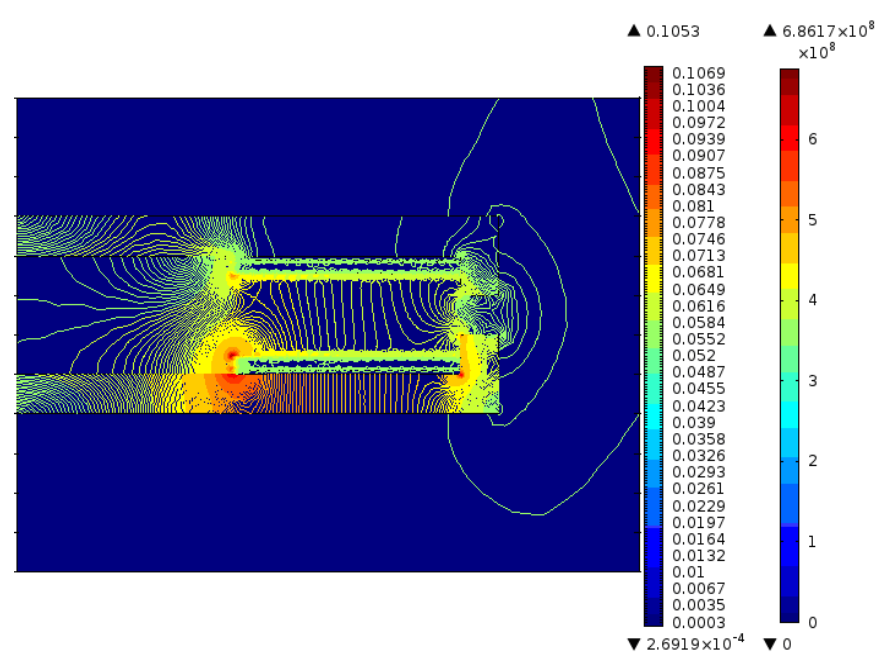

Fig. 9 Magnetic field density and current density of the HFHPD-WPT prototype with U-shaped ferrite cores on both sides, load condition

\section{CONCLUSION}

A U-shaped planar magnetic ferrite is an excellent design for a wireless charging system because of its improvements of a better magnetic flux distribution and this configuration reduces stray fluxes. The near field measurement is done with the help of the near-field scanner and probes. FEM is used to analyze the magnetic flux and eddy current distribution. When the ferrite is used at the secondary side as well as at the primary side, it reduces the flux leakages that go in other direction but this is unable to solve the leakage flux at the edges. As a result, advanced design with a U-shaped magnetic core is able to solve this problem by feeding back the magnetic fluxes into the core and to the secondary side. Consequently, this design is able to solve flux leakages, eddy current and improves power distribution abilities of the system.

\section{REFERENCES}

[1] Fnato,H.,Chiku, Y. and Harakawa, K. "Wireless power distribution with capacitive coupling excited by switched mode active negative capacitor," 2010 International Conference on Electrical Machines and Systems (ICEMS), pp.117-122, Oct.2010.

[2] S. Mohrehkesh and T. Nadeem, "Toward a Wireless Charging for Battery Electric Vehicles at Traffic Intersections," 2011 14th International IEEE Conference on Intelligent Transportation Systems, pp.113-118, Oct.2011.

[3] Pengfei. Li and Bashirullah, R., "A Wireless Power Interface for Rechargeable Battery Operated Medical Implants," IEEE Transactions Circuits and Systems II: Express Briefs, vol.54, no.10, pp.912-916, Oct.2007.

[4] C. Zhu, K. Liu, C. Yu, R. Ma and H. Cheng, " Simulation and Experimental Analysis on Wireless Energy Transfer Based on Magnetic Resonances," IEEE Vehicle Power and Propulsion Conference (VPPC), pp. 1-4,Sept. 2008

[5] W. P. Choi, W. C. Ho, X. Liu and S. Y. R. Hui , "Bidirectional communication techniques for wireless battery charging systems \& portable consumer electronics", IEEE Annual Applied Power Electronics Conference, , pp.2251-2257, Feb. 2010, USA

[6] B. Choi, J. Nho, H. Cha, T. Ahn and S. Choi, "Design and implementation of low-profile contactless battery charger using planar printed circuit board windings as energy transfer device", IEEE Transactions on Industrial Electronics, vol. 51, no. 1, pp. 140-147, Feb. 2004.

[7] Y. Jang and M. M. Jovanovic, "A contactless electrical energy transmission system for portable-telephone battery chargers", IEEE Transactions on Industrial Electronics, vol. 50, no. 3, pp. 520 - 527, Jun. 2003.

[8] C.G. Kim, D.H. Seo, J.-S. You, J.H. Park and B. H. Cho, "Design of a contactless battery charger for cellular phone", IEEE Transactions on Industrial Electronics, vol.48, no.6, pp.1238-1247, Dec.2001.

[9] S. Y. R. Hui and Wong W. C. Ho, "A New Generation of Universal Contactless Battery Charging Platform for Portable Consumer Electronics Equipment," IEEE Trans. On Power Electronics, Vol. 20, No.3, pp.620-627, May.2005.

[10] Q. Yuan, Q. Chen, L. Li, and K. Sawaya, "Numerical Analysis on Transmission Efficiency of Evanescent Resonant Coupling Wireless Power Transfer System," IEEE transactions on antennas and propagation, VOL. 58, no. 5, pp. 1751-1758, May. 2010.

[11] X. Liu and S. Y. R. Hui, "Optimal Design of a Hybrid Winding Structure for Planar Contactless Battery Charger Platform", IEEE Industry Applications Society, vol. 5, pp. 2568 - 2575, Oct. 2006, USA.

[12] S. S. Mohan, M. Hershenson, S. P. Boyd and T. H. Lee, "Simple Accurate Expressions for Planar Spiral Inductances", IEEE Journal of solid-state circuits, vol. 34, No. 10, October 1999.

[13] J. Le-Wei Li, "Wireless Power Transmission: State-of-the-Arts in Technologies and Potential Applications (Invited Paper)," Proceedings of the Asia-Pacific Microwave Conference 2011, pp. 86 - 89, Dec. 2011

[14] B. Yang, F. C. Lee, A. J. Zhang and G. Huang, "LLC resonant converter for front end DC/DC conversion," Applied Power Electronics Conference and Exposition, 2002. APEC 2002. Seventeenth Annual IEEE, Vol. 2, no., pp.1108-1112, August.2002.

[15] C.Panchal, D. Leskarac, J. Lu and S. Stegen, "Investigation of Flux Leakages and EMC Problems in Wireless Charging Systems for EV and Other Mobile Applications, " The Sixth Asia-Pacific Conference on Environmental Electromagnetics Proceedings, pp.301-304, Nov.2012. 